\title{
Image Matching Based on Election Campaign Algorithm
}

\section{Qinghua Xie ${ }^{1, a^{*}}$, Xiangwei Zhang ${ }^{1, b}$, Wenge Lv $^{1, c}$ and Siyuan Cheng ${ }^{1, d}$}

${ }^{1}$ School of Electromechanical Engineering, Guangdong University of Technology, Guangzhou china

axqhcool@163.com, bxwzhang@gdut.edu.cn, 'Ivwenge@163.com, dimdesign@gdut.edu.cn

Keywords: Image matching; Grey scale feature; Rotation invariant feature; Election campaign algorithm

\begin{abstract}
Image matching is widely used in image analysis and computer vision. Traditional Image matching method is to move the template in the reference image pixel by pixel, calculate their gray similarity. It has high computational complexity. If there is a rotation between the template and the reference map, the traditional matching method is difficult to achieve in real time. A method is proposed for matching rotated images based on gray scale feature in this paper, we use Election Campaign Algorithm detect the gray scale feature of points, then rotation invariant feature model, finally using rotation invariant feature model matching point set, image acquisition the translation and rotation parameters. The result of this method is accurate, and the computation complexity is small compared with the traditional correlation matching method, and it is easy to implement in real time.
\end{abstract}

\section{Introduction}

Image matching is a fundamental problem in the field of computer vision, and it is a traditional difficult issue [1]. Detecting the corresponding relationship between two or more images of the same scene from different time, it have different sensors and different visual angles. Because of defects and noise such as the shooting time, shooting angle, natural environment change and sensors variety, the images will be have gray scale level distortion and geometric distortion. It becomes a problem to get a matching algorithm with high accuracy, high matching rate, high speed and strong anti-interference.

Researchers have carried out a lot of research work on image matching, and put forward a lot of methods with good performance. Image matching methods are generally divided into two categories: gray-scale-based matching method and feature-based matching method [2]. Gray-scale-based matching methods, also known as the correlation matching methods, use two dimensional sliding template, the difference of methods is mainly reflected in the choice of the relevant guidelines. Feature-based matching method firstly get the features from the original image, and then match the relationship between the two images. Commonly gray-scale-based methods have better matching results but the computation is bigger [3-5].

In this paper, we use grey scale feature and rotation invariant feature model to match the images. The authors use the election campaign algorithm to find out the most similar position by testing the grey scale feature, and then test the random rotate position of template image to get rotation invariant feature model of matching images [6]. In the optimization process, the similarity position of the image and the template could be find out and the rotation invariant feature model matching begin in the next step.

\section{Grey Scale Feature and Rotation Invariant Feature Model}

Grey scale feature is not sensitive to the deformation and rotation of the target position in the image, and has good stability $[7,8]$.

Set the image grey scale level is $L(O \sim l)$, the general $l$ is 255 . In order to speed up the computing and improve the real-time performance, the grey scale level is mapped to $m$ level, such as $m=8$, which is divided into 8 levels. So, the grey scale level of point is calculated by the Eq. 1 .

$G(r, c)=\left|x^{*} m / l\right|$ 
Corresponding, assuming that the grey scale level of surrounding points at the center point $P(r, c)$, the objective function can be expressed as $S(r, c)$, the sum of levels of points. As is show in Eq. 2.

$$
S(r, c)=\underset{i=1,2, \cdots, n}{\operatorname{sum}}\left(\left|x_{i} * m / l\right|\right)
$$

So, the image matching problem is transformed into the problem of the candidate target position which is the minimum value. When $P(r, c)=S(r, c)-I(r, c)$ is close to 0 , said the target and candidate position are more similar. The target function of optimization algorithm could be like Eq. 3 and Eq. 4 .

$$
\begin{aligned}
& F(r, c)=\underset{i=1,2, \cdots, n}{\operatorname{sum}}\left(\left|x_{i} * m / l\right|\right)-A \\
& A=\operatorname{sum}_{i=1,2, \cdots, n}\left(\left|y_{i} * m / l\right|\right)
\end{aligned}
$$

In the formulas $\mathrm{A}$ is the grey scale feature of the template, $x_{i}$ is the grey scale level of the region in target image position, and $y_{i}$ is the grey scale level of the region in target image position.

After the similar region position is found, rotation invariant feature model is involved to get the rotate angle between the template and the target image.

With rotation invariant feature, image matching problem is transformed into two sets of feature points matching. When the rotation between the two images matched, the corresponding feature point set is bound to rotate. So, it is difficult to match the feature points set.

Matching feature points with rotation is hard to achieve, we use the rotation invariant feature model to test the similarity of the template and the region at the position which find in the peer step. Fig. 1 shows the comparing model of template. The center of template is the solid dot, hollow dots are feature points. The relative position relation in template is used when the same relation is detecting in the target image.

Fig. 2 shows the model of feature point, the sum of gray scale level of surrounding pixels is the numerical character of the feature point. All together the feature points become the rotation invariant feature model. First, rotate the model of template, than test the similarity of the rotated template and target region in the image. Such as the matching test is show in Fig. 3.

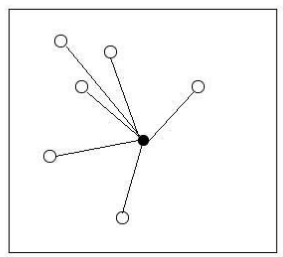

Figure 1. Comparing Model of Template

\begin{tabular}{|l|l|l|}
\hline 1 & 2 & 3 \\
\hline 4 & 5 & 6 \\
\hline 7 & 8 & 9 \\
\hline
\end{tabular}

Figure 2. Grey Scale Model for Matching Feature Point

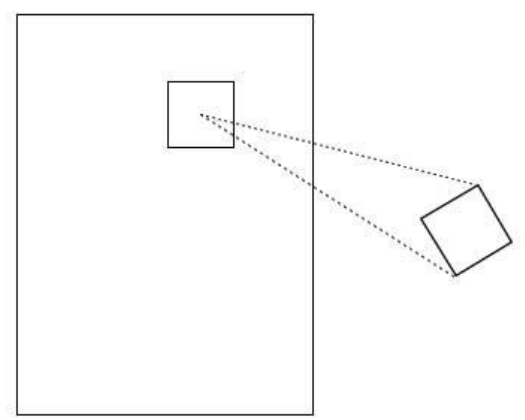

Figure 3. Rotated Template Matching 


\section{Election Campaign Algorithm}

Election campaign algorithm (ECA) is a new optimization algorithm simulating election process. Election candidates always pursue the maximum support from voters by means of various election behaviors $[6,9]$. They influence the voters round them, the voter's support is in proportion to the distance between the candidates and the voters. The voter will vote to the candidate which he pefer the most. The sum of location coordinates of every voters supported the candidate powered by its contribution is a new location coordinates, which is named support focus, it is the next position of the candidate. Such computational cycle is done continually until the function finds the position of the highest support, which is the global solution of the optimization problems [10].

Set the Algorithm Parameters. Main parameters of Election campaign algorithm are:

- The number of candidates.

- The number of voters.

- The number of floating voters.

- Target accuracy.

Generate the Candidates and Calculate the Prestige of Them. Generated the define number of candidates in feasible solution field on the uniform distribution. Use the objective function to calculate the prestige of each candidate.

Generate the Voters. The uniform distribution is employed to generate the voters in feasible solution field.

Compute the Investigate Mean Square of Candidates. Higher prestige of a candidate, smaller the mean square deviation of local voters, so that ECA is able to converge to local optimization solution rapidly and steadily.

Calculate the Supported of the Voters. The support of a voter is proportional to his prestige, and then the proportional constant will be reduced, so the prestige of a voter can used to denote the support of a voter directly. A voter may be influenced by several candidates; the voter should distribute his support to candidates proportionally on the magnitude of effect from candidate to voter.

Find the Support Focus of Candidates. A new position coordinate will achieve by means of summing the products of the support from the voters to the candidate and the position coordinate of the voters. It is named the support focus.

The support focus of a candidate is obtained by investigating, which depends on those voters whose distances to the candidate are nearer and the prestige is higher relatively. The next post of the candidate should be the support focus, where the candidate will have the higher support.

Calculate the Prestige of the Candidates and Compare the Prestige of the Voters with the Candidates. In order to jump out of local optimization solution and increase search rate, the prestige of candidates are compared to that of voters, if the prestige of a voter is higher than that of a candidate, the voter with higher prestige will substitute for the candidate and the that candidate of lower prestige will be eliminated in election.

Check Whether the Condition Is Reach. Check whether the condition is reach, otherwise return to step B to execute the period. Here, the condition could be the relative position relation in target image. Do that circularly until the highest support is not change.

$$
\left|\operatorname{sum}_{i=1,2, \cdots, 9} S_{c}\left(r_{i}^{r}, c_{i}^{r}\right)-\operatorname{sum}_{i=1,2, \cdots, 9} S_{t}\left(r_{i}, c_{i}\right)\right|<\xi
$$

$S_{c}$ represents the position relation of template, $S_{t}$ represents the position relation of target image region. Feature points of template $P_{r}\left(r_{i}, c_{i}\right)$ and points of target image region $P\left(r_{i}, c_{i}\right)$ have the same relative position relation. The right parameter on Eq. 5 is a very small number. Random rotation is set in the template as voters in ECA and the support focus in the previous ECA cycle is position to test more similar position. If the highest support is not change, the computation stop. When ECA stop, the candidate image is similar if Eq. 5 is workable. 


\section{Experiment and Result}

In the image of Fig. 4, the first image is the target image, other is the template image.

The similar position in target image is easily found and random angles are set to test the relation of template and the region of target image. Using election campaign algorithm, the number of candidates define as 5 , the number of voters is 10 , the number of floating voters is 5 , and the target accuracy is set as 2.5. The experiment get the similar position first then find the angle of template most similar with the target image. Fig. 5 shows the results of the whole process of the algorithm run in both step and get the position and angle which the template match most likely.
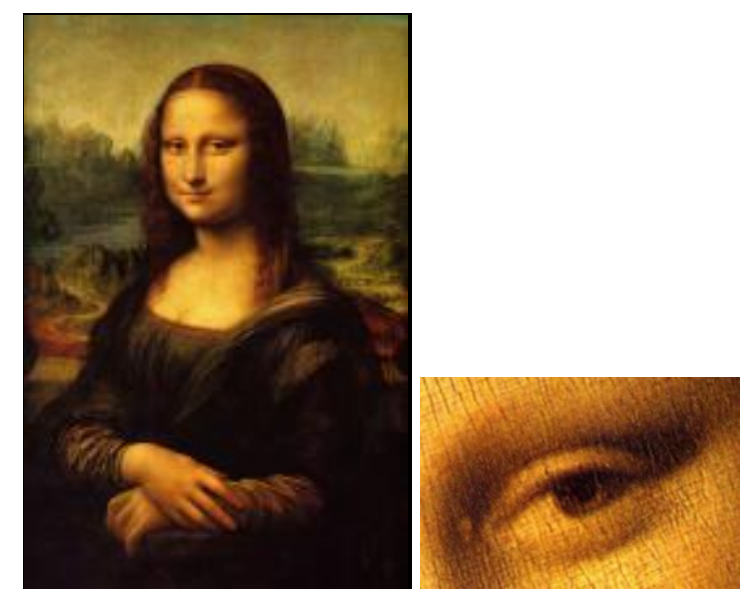

Figure 4. Target Image and Enlarged Template
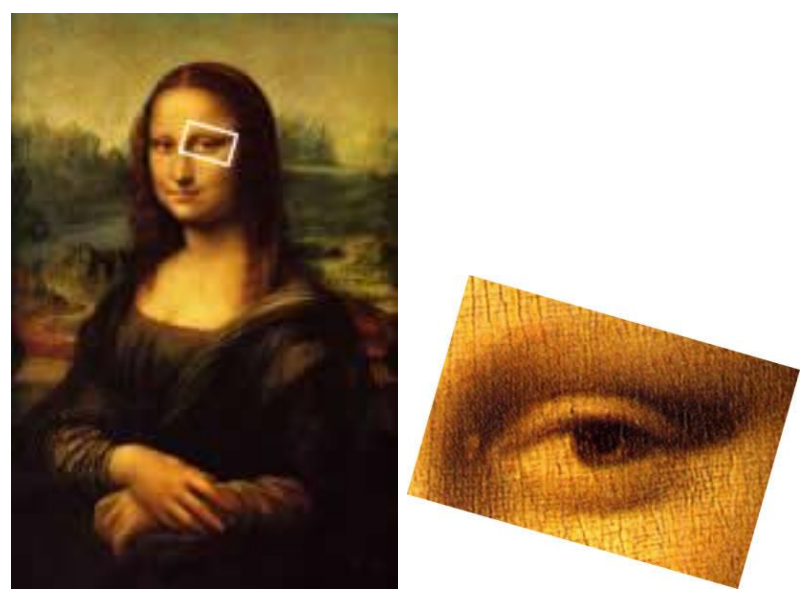

Figure 5. Results of ECA Image Matching

\section{Conclusions}

With the method of comparing the sum of gray scale feature levels, the similar position of image can be detected. Election campaign algorithm optimization program detect the position first, then in second step the angle of template. In both step, election campaign algorithm has the advantages of parallel computing and good performance to avoid the solution trapped in local optima. This is suitable method to find the maximum of grey scale feature sum and the relation between two set of numerical value.

\section{References}

[1] Du-Ming T, Ya-Hui T, Rotation-invariant pattern matching with color ring-projection, Pattern Recognition, 2002, 35(3), pp. 131-141.

[2] Balslev I, Doring K, Erikson R D, Weighted central moments in pattern recognition, Pattern Recognition, 2000, 21(4):381-384

[3] Prieto M.S., Allen A.R., A similarity metric for edge images, IEEE Trans. Pattern Analysis and Machine Intelligence, 2003, 25(10), pp. 1265-1273.

[4] Schiele B., Crow ley J.L., Recognition without Correspondence Using Multidimensional Receptive Field Histograms, Int'1 Journal of Computer Vision, 2000, 36(1), pp. 31-50.

[5] Wang Y.T., Zhang D.Z., Tian J.W., Topological clustering and its application for discarding wide-baseline mismatches, Optical Engineering, 2008, 47(4).

[6] Wenge Lv, Qinghua Xie, Zhiyong Liu, Deyuan Li, et al., Verifying Election Campaign Optimization Algorithm by Several Benchmarking Functions, Lecture Notes in Computer Science, 6146 LNCS, 2, pp. 582-57.

[7] Gu Xiaodong, Yang Cheng, Application of new color similarity measurement method in image retrieval, Chinese Journal of Scientific Instrument, 2014, 35(10), pp. 2286-2292. 
[8] Mojsilovic A, Hu J.Y., Soljanin E, Extraction of perceptually important colors and similarity measurement for image matching, retrieval and analysis , IEEE Trans on Image Processing, 2002, 11(11), pp. 1238-1248.

[9] Chunhua He, Wenge Lv, Xiangwei Zhang, et al., Election-survey algorithm and its application in global optimization problem of function, Computer Engineering and Applications, 2010, 46(11), pp. 196-199.

[10] Wenge Lv, Chunhua He, DeyuanLi, et al., Election Campaign Optimization Algorithm, Procedia Computer Science, 1, pp. 1371-1380. 